\title{
PERMANENCE OF IDENTITIES ON ALGEBRAS
}

\author{
M. N. BLEICHER, HANS SCHNEIDER and R. L. WILSON ${ }^{1}$ )
}

\section{§1. Introduction}

In our previous paper [1] we investigated under what circumstances the identities of a partially ordered (universal) algebra remain valid when the algebra is completed to a suprema preserving superior completion $([1],(2.6),(3.7),(5.11))$. In general we use the terminology and notation of [1], with some minor exceptions that will be noted later. We there showed that if both sides of an identity contain no repeated variables then the identity remains valid under the completion. Gautam [7] handled the unordered case of the problem in [1] where $T$ is the complex algebra including the empty set, which causes certain changes. Fuchs' work [5], [6] also has considerable overlap with [1], although our papers were written independently. (See also Clifford [3].) In this paper we consider the converse problem; namely, if the identities on a (finitary) algebra and its completion are the same what can be said about the identities of the original algebra. From another point of view we give conditions under which the identities on an algebra can be determined by looking at the identities on appropriate sub algebras. We maintain the former point of view in this paper.

In $\S 2$ we introduce the notions of split words and split pairs of words and give a quasi ordering to the words of a free algebra (called polynomial algebra by Grätzer [8]), in which the maximal words are the split words except when the cardinality of the generating set is finite and the word is long. At the suggestion of the referee we now use the term 'linear' where 'split' was used in [1].

In $\S 3$, we prove two technical lemmas about homomorphisms and suprema of a certain special collection of points in the completion $T$ of an algebra $S$.

In $\S 4$, we prove the main results. These are simplest to describe when $S$ is trivially ordered (unordered) and free over itself with an infinity of generators and $T$ is the complex algebra. We show (4.17) that the identities on $S$ and $T$ are the same if and only if the identities on $S$ considered as a fully invariant congruence on a free infinitely generated (polynomial) algebra are generated by linear pairs of words. As a corollary we obtain (4.10) that an equational class is closed under taking complex algebras if and only if the smallest fully invariant congruence on an infinitely generated free algebra containing the defining identities is generated by pairs of linear words. When the order is non-trivial or the number of free generators of $S$ is finite, then more

1) We wish to thank the National Science Foundation for partial support under grant GP-9564 and GP-6826 during the preparation of this paper.

Presented by G. Grätzer. Received February 23, 1972. Accepted for publication in final form July 9, 1973. 
technical hypotheses are given which are sometimes weaker and sometimes stronger, (4. 15) and (4. 16). These hypotheses balance off the freedom given by more generators against the restriction required by the ordering. Various examples are given to show on the one hand that the hypotheses are satisfiable in a non-trivial way and, on the other hand, that if one of them is omitted the conclusion need not hold.

In $\S 5$, we give a sufficient condition for a particular identity to remain valid in a completion (5.3). We show (5.2) that the condition is equivalent to a condition relied upon in [1]. These conditions are not necessary for the identity to remain valid.

All algebras considered in this paper are finitary.

We take this opportunity to point out an omission in $\$ 6$ of [1], in which the hypothesis that the algebra be finitary was not stated. The proofs use that hypothesis, and examples in [2] show that it is in fact necessary.

For a fuller version of this paper containing additional proofs see [2] and for some other details see Wilson [9].

The authors wish to thank G. Gratzer for his helpful suggestions in matters of presentation.

\section{§2. Linear words in free algebras}

\section{(2.1) Definitions and Remarks}

(2.1.1) By $\langle\Omega, X\rangle$ we denote the free $\Omega$-algebra with generator set $X$, while $(\Omega, S)$ denotes an $\Omega$-algebra with $S$ as the set of elements. For each word $w$ in the free algebra $\langle\Omega, X\rangle$ and for each $x \in X$, we denote by $\mu_{x}(w)$ the multiplicity of $x$ in $w$, i.e., the number of occurrences of $x$ in $w$.

(2.1.2) Since $\mu_{x}(w)>0$ for only a finite number of $x \in X$, we may write

$$
\mu(w)=\sum\left\{\mu_{x}(w): x \in X\right\} .
$$

(2.1.3) The support of $w$ is defined to be

and

$$
\Gamma(w)=\left\{x \in X: \mu_{x}(w)>0\right\}
$$

$$
\gamma(w)=|\Gamma(w)| .
$$

(2.1.4) Intuitively $\mu(w)-\gamma(w)$ is the number of repetitions of variable symbols in $w$, e.g., in the free groupoid $\langle\cdot, X\rangle$ we have

$$
\begin{gathered}
\mu\left(x^{2} y\right)=3, \\
\gamma\left(x^{2} y\right)=2, \\
\mu\left(x^{2} y\right)-\gamma\left(x^{2} y\right)=1 \quad(x \text { is repeated once }) .
\end{gathered}
$$

(2.1.5) A word $w \in\langle\Omega, X\rangle$ is called linear if $\mu_{x}(w) \leqslant 1$, for each $x \in X$. 
(2.1.6) For all $w \in W, \mu(w) \geqslant \gamma(w)$ and the word $w$ is linear if and only if

$$
\mu(w)=\gamma(w) .
$$

(2.1.7) By convention $\phi$ will always denote a substitution of $X$ into $X$. As is observed in [1], every substitution $\phi: X \rightarrow X$ has a unique extension from $\langle\Omega, X\rangle$ into $\langle\Omega, X\rangle$. We denote this extension again by $\phi$.

\section{(2.2) More definitions and remarks}

(2.2.1) Let $w, w^{\prime} \in\langle\Omega, X\rangle$. We define $w^{\prime} \geqslant w$ if there exists a substitution $\phi: X \rightarrow X$ such that $\phi\left(w^{\prime}\right)=w$.

(2.2.2) It is easy to see that $\geqslant$ is a quasi ordering.

(2.2.3) If $w, w^{\prime} \in\langle\Omega, X\rangle$ and both $w^{\prime}>w$ and $w>w^{\prime}$, we write $w \sim w^{\prime}$. (Clearly, $\sim$ is an equivalence relation on $\langle\Omega, X\rangle$.) If $w^{\prime} \geqslant w$, but not $w \geqslant w^{\prime}$, we write $w^{\prime}>w$.

(2.2.4) If $w \in W \subseteq\langle\Omega, X\rangle$, but there is no $w^{\prime} \in W$ such that $w^{\prime}>w$, we call $w$ maximal in $W$.

(2.2.5) Given $w, w^{\prime} \in\langle\Omega, X\rangle$, there exists a permutation $\pi$ of $X$ such that $\pi\left(w^{\prime}\right)=w$, if and only if $w^{\prime} \sim w$. Further $w^{\prime} \geqslant w$, if and only if $w=\phi \pi\left(w^{\prime}\right)$, where $\pi$ is a permutation on $X$, and $\phi$ is an idempotent substitution of $X$ into itself.

(2.2.6) If $w^{\prime} \geqslant w$ then

$$
\begin{aligned}
& \mu\left(w^{\prime}\right)=\mu(w), \\
& \gamma\left(w^{\prime}\right) \geqslant \gamma(w) .
\end{aligned}
$$

(2.2.7) Let $w^{\prime} \geqslant w$. Then $w^{\prime}>w$ if and only if

$$
\gamma\left(w^{\prime}\right)>\gamma(w)
$$

A formal inductive definition of $\mu_{x}(w)$ and proof of the above remarks can be found in Wilson [9].

(2.3) Definitions and remarks

(2.3.1) Let $\left(w_{1}, w_{2}\right) \in\langle\Omega, X\rangle^{2}$. For $x \in X$, we define

and

$$
\mu_{x}\left(w_{1}, w_{2}\right)=\max \left(\mu_{x}\left(w_{1}\right), \mu_{x}\left(w_{2}\right)\right)
$$

$$
\mu\left(w_{1}, w_{2}\right)=\sum\left\{\mu_{x}\left(w_{1}, w_{2}\right): x \in X\right\}
$$

(2.3.2) We define

$$
\Gamma\left(w_{1}, w_{2}\right)=\Gamma\left(w_{1}\right) \cup \Gamma\left(w_{2}\right)
$$

and

$$
\gamma\left(w_{1}, w_{2}\right)=\left|I\left(w_{1}, w_{2}\right)\right| .
$$


(2.3.3) A pair $\left(w_{1}, w_{2}\right) \in\langle\Omega, X\rangle^{2}$ is called linear if and only if each $w_{1}$ and $w_{2}$ is linear.

(2.3.4) A pair $\left(w_{1}, w_{2}\right)$ is linear if and only if

(2.3.5) Evidently

$$
\mu\left(w_{1}, w_{2}\right)=\gamma\left(w_{1}, w_{2}\right)
$$

and

$$
\begin{aligned}
& \mu\left(w_{1}, w_{2}\right) \leqslant \mu\left(w_{1}\right)+\mu\left(w_{2}\right), \\
& \gamma\left(w_{1}, w_{2}\right) \leqslant \gamma\left(w_{1}\right)+\gamma\left(w_{2}\right),
\end{aligned}
$$

$$
\gamma\left(w_{1}, w_{2}\right) \leqslant \mu\left(w_{1}, w_{2}\right)
$$

(2.4) Yet more definitions and remarks

(2.4.1) In analogy with $w^{\prime} \geqslant w$, we define for $\left(w_{1}, w_{2}\right),\left(w_{1}^{\prime}, w_{2}^{\prime}\right) \in\langle\Omega, X\rangle^{2}$, that $\left(w_{1}^{\prime}, w_{2}^{\prime}\right) \geqslant\left(w_{1}, w_{2}\right)$ if there exists $\phi: X \rightarrow X$ such that $\phi\left(w_{1}^{\prime}\right)=w_{1}$ and $\phi\left(w_{2}^{\prime}\right)=w_{2}$.

(2.4.2) Evidently $\left(w_{1}^{\prime}, w_{2}^{\prime}\right) \geqslant\left(w_{1}, w_{2}\right)$ implies $w_{1}^{\prime} \geqslant w_{1}$ and $w_{2}^{\prime} \geqslant w_{2}$, but not conversely.

(2.4.3.) We define $\left(w_{1}^{\prime}, w_{2}^{\prime}\right) \sim\left(w_{1}, w_{2}\right),\left(w_{1}^{\prime}, w_{2}^{\prime}\right)>\left(w_{1}, w_{2}\right)$ and $\left(w_{1}, w_{2}\right)$ is maximal in $W \subseteq\langle\Omega, X\rangle^{2}$ as in (2.2.3) and (2.2.4).

(2.4.4) If $\left(w_{1}^{\prime}, w_{2}^{\prime}\right) \geqslant\left(w_{1}, w_{2}\right)$ then

and

$$
\mu\left(w_{1}^{\prime}, w_{2}^{\prime}\right) \geqslant \mu\left(w_{1}, w_{2}\right)
$$

$$
\gamma\left(w_{1}^{\prime}, w_{2}^{\prime}\right) \geqslant \gamma\left(w_{1}, w_{2}\right)
$$

(2.4.5) Let $\left(w_{1}^{\prime}, w_{2}^{\prime}\right) \geqslant\left(w_{1}, w_{2}\right)$. Then

if and only if

$$
\left(w_{1}^{\prime}, w_{2}^{\prime}\right)>\left(w_{1}, w_{2}\right)
$$

$$
\gamma\left(w_{1}^{\prime}, w_{2}^{\prime}\right)>\gamma\left(w_{1}, w_{2}\right)
$$

(2.5) LEMMA. Every ascending chain of pairs in $\langle\Omega, X\rangle^{2}$ is finite.

Proof. Omitted.

(2.6) LEMMA. Let $W \subseteq\langle\Omega, X\rangle^{2}$. Then for each $\left(v_{1}, v_{2}\right) \in W$ there exists a pair $\left(w_{1}, w_{2}\right)$ such that $\left(v_{1}, v_{2}\right) \leqslant\left(w_{1}, w_{2}\right)$ and $\left(w_{1}, w_{2}\right)$ is maximal in $W$.

This is an immediate consequence of (2.5).

(2.7) LEMMA. Let $\left(w_{1}, w_{2}\right) \in\langle\Omega, X\rangle^{2}$ and suppose $\mu\left(w_{1}, w_{2}\right) \leqslant|X|$. Then there exists a linear pair $\left(w_{1}^{\prime}, w_{2}^{\prime}\right) \in\langle\Omega, X\rangle^{2}$ such that $\left(w_{1}^{\prime}, w_{2}^{\prime}\right) \geqslant\left(w_{1}, w_{2}\right)$.

Proof. Omitted. 
(2.8) COROLLARY. Let $w \in\langle\Omega, X\rangle$ and suppose $\mu(w) \leqslant|X|$. Then there exists a linear word $w^{\prime} \in\langle\Omega, X\rangle$ such that $w^{\prime} \geqslant w$.

Proof. Put $\left(w_{1}, w_{2}\right)=(w, w)$ in $(2.7)$.

\section{(2.9) Remarks}

It is easy to show that if $w \in\langle\Omega, X\rangle$ is linear, then $w$ is maximal in $\langle\Omega, X\rangle$. If $w$ is maximal and $\gamma(w)<|X|$, then $w$ is linear. Also if $w$ is linear, and $w^{\prime} \geqslant w$, then $w^{\prime}$ is linear. However, there exists linear pairs $\left(w_{1}, w_{2}\right)$ which are not maximal in $\langle\Omega, X\rangle$. For example, $(x y, y x)$ is linear in the free groupoid $(\cdot, X\rangle$, but not maximal.

\section{§3. $\psi$ and $\psi$}

We now prove a theorem which describes the effect of a homomorphism into a suprema preserving ([1], (5.11)) superior completion ([1], (2.6) and (3.7)) of a partially ordered algebra $(\Omega, S)$. If $\omega \in \Omega$ is $n$-ary and $t_{i} \in T, i=0,1, \ldots, n-1$, we here write $\omega\left(t_{0}, \ldots, t_{n-1}\right)$ where in [1] we wrote $* \omega\left(t_{0}, \ldots, t_{n-1}\right)$ or $* \omega(\mathrm{t})$.

\section{(3.1) Convention}

Henceforth $\theta\left(\right.$ or $\left.\theta_{i}\right)$ will denote a homomorphism from $\langle\Omega, X\rangle$ to $(\Omega, S)$.

(3.2) THEOREM. Let $(\Omega, S)$ be a partially ordered universal algebra, and let $(\Omega, T)$ be a suprema preserving superior completion of $(\Omega, S)$. Let $\psi$ be a homomorphism of $\langle\Omega, X\rangle$ into $(\Omega, T)$. Let $w, w^{\prime} \in\langle\Omega, X\rangle$, where $w^{\prime}$ is linear, $\phi: X \rightarrow X$ and $\phi\left(w^{\prime}\right)=w$. If $\Gamma\left(w^{\prime}\right) \subseteq Y \subseteq X$, and, for all $x \in Y, A(x)$ is a subset of $S$ such that $\sup _{r} A(x)=\psi \phi(x)$, then

$$
\psi(w)=\sup _{T}\left\{\theta\left(w^{\prime}\right): \theta(x) \in A(x), \text { if } \quad x \in Y\right\}
$$

Proof. The proof is by induction on depth $w^{\prime}$ (see [1], (5.25), and $\S 7$ for the properties of depth). If $w^{\prime}=\omega \in \Omega_{0}$, the result is obvious. If $w^{\prime}=y \in X$, then $w=z \in X$ and $\phi(y)=z$. Hence

$$
\sup _{T}\{\theta(y): \theta(x) \in A(x), \quad \text { if } \quad x \in Y\}=\sup _{T} A(y)=\psi \phi(y)=\psi(z) .
$$

Hence the result is true if depth $w^{\prime}=1$. Let depth $w^{\prime}>1$, and suppose the result is true for all words of depth less than depth $w^{\prime}$. Then $w^{\prime}$ must have the form

$$
w^{\prime}=\omega\left(w_{0}^{\prime}, \ldots, w_{i}^{\prime}, \ldots, w_{n-1}^{\prime}\right)
$$

Since $w=\phi\left(w^{\prime}\right)$, we have

$$
w=\omega\left(w_{0}, \ldots, w_{i}, \ldots, w_{n-1}\right)
$$


where $w_{i}=\phi\left(w_{i}^{\prime}\right), i=0, \ldots, n-1$.

$$
\begin{aligned}
\psi(w) & =\psi\left(\omega\left(\ldots, w_{i}, \ldots\right)\right) \\
& =\omega\left(\ldots, \psi\left(w_{i}\right), \ldots\right) \\
& =\omega\left(\ldots, \sup _{T}\left\{\theta_{i}\left(w_{i}^{\prime}\right): \theta_{i}(x) \in A(x) \text { for } \quad x \in Y\right\}, \ldots\right) \\
& =\sup _{T}\left\{\omega\left(\ldots, \theta_{i}\left(w_{i}^{\prime}\right), \ldots\right): \theta_{i}(x) \in A(x) \text { if } x \in Y, i=0, \ldots, n-1\right\}
\end{aligned}
$$

since $w_{i}^{\prime}$ is linear, $\Gamma\left(w_{i}^{\prime}\right) \subseteq Y$ and $(\Omega, T)$ is suprema preserving. But the supports $\Gamma\left(w_{i}^{\prime}\right), i=0, \ldots, n-1$ are pairwise disjoint, since $w^{\prime}$ is linear. Hence given $\theta_{0}, \ldots, \theta_{n-1}$ there exists a substitution $\theta$ such that

$$
\theta(x)=\theta_{i}(x) \text { if } x \in \Gamma\left(w_{i}\right)
$$

and

$$
\theta(x)=\theta_{0}(x) \text { otherwise }
$$

It follows that

$$
\begin{aligned}
\psi(w) & =\sup _{T}\left\{\omega\left(\ldots, 0\left(w_{i}^{\prime}\right), \ldots\right): \theta(x) \in A(x) \text { if } \quad x \in Y\right\} \\
& =\sup _{T}\left\{\theta\left(\omega\left(\ldots, w_{i}^{\prime}, \ldots\right)\right): \theta(x) \in A(x) \text { if } x \in Y\right\} \\
& =\sup _{T}\left\{\theta\left(w^{\prime}\right): \theta(x) \in A(x) \text { if } x \in Y\right\} .
\end{aligned}
$$

The theorem follows by induction.

\section{(3.3) Example}

Theorem (3.2) need not hold if $(\Omega, T)$ is not suprema preserving as an extension of $(\Omega, S)$. As an example, let $S=\left\{a_{1}, a_{2}, b_{1}, b_{2}, 0\right\}$ with $a_{1}>a_{2}>0, b_{1}>b_{2}>0$, (see Fig. 1) and define a binary operation by $a_{1}^{2}=a_{2}, b_{1}^{2}=b_{2}$, and all other products are 0 . Let $T=S \cup\{u\}$, where $u>s$ for $s \in S$. Then $\left(s^{2}\right) s=\{0\}$, but in $(\Omega, T)$,

$$
u^{2}=\sup _{T}\left\{s_{1}, s_{2}: s_{i} \in S\right\}=u,
$$

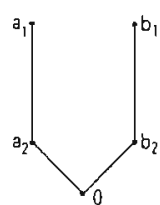

$S$

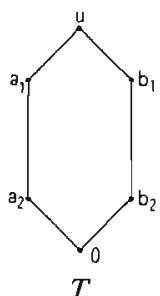

$T$

Fig. 1 
so $\left(u^{2}\right) u=u$. Let $X=\left\{x_{1}, x_{2}, x_{3}\right\}$ and let $\langle\cdot, X\rangle$ be a free groupoid. Let $\psi: X \rightarrow(\cdot, T)$ such that $\psi(x)=u$ for all $x \in X$. Then $\psi\left(\left(x^{2}\right) x\right)=u$, but if

$$
\begin{aligned}
& \phi\left(x_{1}\right)=\phi\left(x_{2}\right)=\phi\left(x_{3}\right)=x \\
& \sup _{T}\left\{\theta\left(\left(x_{1} x_{2}\right) x_{3}\right)\right\}=0 \neq u
\end{aligned}
$$

In this example $Y=X$ and $A(x)=S$, for all $x \in X$.

If $\psi$ is a homomorphism of $\langle\Omega, X\rangle$ into $(\Omega, T)$ let $\psi$ be the mapping of $\langle\Omega, X\rangle$ into $(\Omega, T)$ defined in [1] (5.23), viz.

$$
\psi(w)=\sup _{T}\{\theta(w): \theta(x) \leqslant \psi(x) \text { for all } x \in X\}
$$

More generally:

(3.4) PROPOSITION. Let $(\Omega, T)$ be a suprema preserving superior extension of $(\Omega, S)$. Let $\psi$ be a homomorphism of $\langle\Omega, X)$ into $(\Omega, T)$. If $w \in\langle\Omega, X\rangle, \Gamma(w) \subseteq Y \subseteq$ $\subseteq X, A(y) \subseteq S$ for $y \in Y$ and $\sup _{T} A(y)=\psi(y)$ for all $y \in Y$ then

$$
\Psi(w)=\sup _{T}\{\theta(w): \theta(y) \in A(y), \text { for } y \in Y\}
$$

The proof is along the same general lines as that of (3.2).

\section{§4. Permanence of identities}

For the sake of completeness, we include the following definitions.

\section{(4.1) Definitions}

(4.1.1) Let $(\Omega, S)$ be an algebra and let $\langle\Omega, X\rangle$ be a free algebra. Let $\left(w_{1}, w_{2}\right) \epsilon$ $\epsilon\langle\Omega, X\rangle^{2}$. Then $\left(w_{1}, w_{2}\right)$ is an identity on $(\Omega, S)$ from $\langle\Omega, X\rangle$ if for all substitutions $\theta: X \rightarrow(\Omega, S), \theta\left(w_{1}\right)=\theta\left(w_{2}\right)([4]$, p. 162$)$.

(4.1.2) The set of all identities on $(\Omega, S)$ from $\langle\Omega, X\rangle$ will be denoted by $\operatorname{Id}(S, X)$, or Id $(S)$ where no confusion is likely to arise. We may write $w_{1} \equiv w_{2}$ for $\left(w_{1}, w_{2}\right) \in$ $\in \operatorname{Id}(S, X)$.

(4.1.3) As usual a non-empty subset $\mathscr{Q}$ of $(\Omega, A) \times(\Omega, A)$ is called a congruence if it is an equivalence relation and in addition for each $\omega \in \Omega$ and $\left(u_{i}, v_{i}\right) \in \mathscr{Q}$ for $i=0, \ldots$, $n-1$, we have $\left(\omega\left(u_{0}, \ldots, u_{n-1}\right), \omega\left(v_{0}, \ldots, v_{n-1}\right)\right) \in \mathscr{Q}$.

(4.1.4) A congruence 2 on an algebra $(\Omega, A)$ will be called a fully invariant congruence if $\left(u_{1}, u_{2}\right) \in \mathscr{Q}$ implies $\left(\lambda\left(u_{1}\right), \lambda\left(u_{2}\right)\right) \in \mathscr{Q}$ for each endomorphism $\lambda$ of $(\Omega, A)$. (Grätzer [8], p. 70.)

(4.2) LEMMA. The set $\operatorname{Id}(S, X)$ is a fully invariant congruence on $\langle\Omega, X\rangle$. 
Proof. Omitted.

The following illustrates the intuitive content of Lemma (4.2). Let $(\cdot, S)$ be a groupoid satisfying the identity $(x y) z \equiv x(y z)$ from $\langle\Omega, X\rangle$. Then $(\cdot, S)$ also must satisfy such identities as $(x y) x \equiv x(y x), x^{2} y \equiv x(x y)$, and $((x y) z) x^{2} \equiv(x y)(z x)^{2}$, etc.

If the algebra $(\Omega, S)$ is represented as a factor algebra of a free algebra, then the maximal fully invariant congruence contained in the kernel is simply $\operatorname{Id}(S, X)$. More precisely,

(4.3) LEMMA. Let $\mathscr{Q}$ be a congruence on $\langle\Omega, X\rangle$. If $(\Omega, S)=\langle\Omega, X\rangle / \mathscr{Q}$ then $\operatorname{Id}(S, X)$ is the unique maximal fully invariant congruence contained in $\mathscr{Q}$.

Proof. Omitted.

(4.4) DEFINITIONS. Let $n$ be a cardinal. An algebra $(\Omega, S)$ is called free over itself on $n$ generators, if and only if there is a subset $G$ of $S$, with $|G|=n$, such that $G$ generates $(\Omega, S)$ and such that every mapping of $G$ into $S$ can be extended to a homomorphism from $(\Omega, S)$ to $(\Omega, S)$.

The equivalence of (1) and (2) of our next theorem is to be found in Grätzer [8], except that in that result the cardinality of the generating set is not mentioned.

(4.5) THEOREM. Let $(\Omega, S)$ be an algebra with more than one element. Then the following are equivalent:

(1) $(\Omega, S)$ is free over itself on $n$ generators;

(2) there exists $X$ with $|X|=n$, such that

$$
(\Omega, S) \cong\langle\Omega, X\rangle / \operatorname{Id}(S, X)
$$

(3) there exists $X$, with $|X|=n$ and a fully invariant congruence $\mathscr{Q}$ on $\langle\Omega, X\rangle$, such that

$$
(\Omega, S) \cong\langle\Omega, X\rangle / \mathscr{Q}
$$

Proof. Omitted.

We observe that if $(\Omega, S)$ has just one element, then $(\Omega, S)$ is free over itself with one generator, and

$$
(\Omega, S)=\langle\Omega,\{x\}\rangle / \mathscr{Q}, \text { where } \mathscr{Q}=\langle\Omega,\{x\}\rangle .
$$

However, if $|X|=n>1$, and $\mathscr{Q}=\langle\Omega, X\rangle$, then $(\Omega, S)$ has one element; hence the restriction $|(\Omega, S)|>1$ in $(4.5)$.

(4.6) LEMMA. Let $\mathscr{Q}$ be a fully invariant congruence on $\langle\Omega, X\rangle$ and let $Q$ be the subset of $\mathscr{Q}$ consisting of all $\left(w_{1}^{\prime}, w_{2}^{\prime}\right) \in \mathscr{Q}$ which are maximal in $\mathscr{Q}$ under $<$. Then $Q$ generates 2 as a fully invariant congruence. 
Proof. Omitted.

We observe that in general a proper subset of the set of elements maximal in $\mathscr{Q}$ suffices to generate $\mathscr{Q}$.

We now begin detailed investigation of the properties that force identities to remain true for suprema preserving superior completions.

(4.7) DEFINITION. Let $(\Omega, T)$ be a suprema preserving superior completion of the partially ordered algebra $(\Omega, S)$. We call $s \in T$ isolated in $S$ by $T$ if $s \leqslant \sup _{T} B$, where $B \subseteq S$, implies that $s \in B$.

(4.8) Remarks

(4.8.1) Since $T$ is a superior extension of $S, t \in T \backslash S$ implies that $t=\sup _{T}\{s \in S$ : $s \leqslant t\}$. Hence if $s \in T$ and $s$ is isolated in $S$ by $T$ then $s \in S$.

(4.8.2) Evidently, every element $s$ isolated in $S$ by $T$ is maximal in $S$.

(4.8.3) Let $T=\mathscr{S}(S)$. Then $s \in S$ is isolated in $S$ by $\mathscr{S}(S)$ if and only if $s$ is maximal in $S$.

(4.8.4) $S$ is trivially ordered, if and only if every $s \in S$ is isolated in $S$ by $\mathscr{S}(S)$.

(4.9) LEMMA. Let $\eta$ be a homomorphism of $\langle\Omega, X\rangle$ into the partially ordered algebra $(\Omega, S)$, and let $(\Omega, T)$ be a suprema preserving superior completion of $(\Omega, S)$. Let $w_{1}^{\prime}, w_{2}^{\prime}$ be linear words in $\langle\Omega, X\rangle$ with $\eta\left(w_{1}^{\prime}\right)$ isolated in $S$ by $T$. Let $\phi: X \rightarrow X$ and $\phi\left(w_{i}^{\prime}\right)=w_{i}, i=1,2$. If $\psi$ is a homomorphism of $\langle\Omega, X\rangle$ into $(\Omega, T)$ satisfying for all $x \in Y=\Gamma\left(w_{1}^{\prime}\right) \cup \Gamma\left(w_{2}^{\prime}\right)$

$$
\psi \phi(x)=\sup _{T} \eta \phi^{-1} \phi(x)
$$

then $\psi\left(w_{1}\right)=\psi\left(w_{2}\right)$ implies that there exists $w_{2}^{\prime \prime} \in\langle\Omega, X\rangle$ such that

$$
\begin{aligned}
w_{2}^{\prime} & \geqslant w_{2}^{\prime \prime}, \\
\left(w_{1}^{\prime}, w_{2}^{\prime \prime}\right) & \geqslant\left(w_{1}, w_{2}\right),
\end{aligned}
$$

and

$$
\eta\left(w_{1}^{\prime}\right)=\eta\left(w_{2}^{\prime \prime}\right)
$$

Proof. Let $A(x)=\eta \phi^{-1} \phi(x)$, for $x \in X$. By (3.2), we have for $i=1,2$,

$$
\psi\left(w_{i}\right)=\sup _{T}\left\{\theta\left(w_{i}^{\prime}\right): \theta(x) \in A(x) \text { for } x \in Y^{\prime}\right\} .
$$

Since $\eta(x) \in A(x)$ for all $x \in X$, we have

$$
\begin{aligned}
\eta\left(w_{1}^{\prime}\right) \leqslant \sup _{T}\left\{\theta\left(w_{1}^{\prime}\right): \theta(x) \in A(x), \text { for } x \in Y\right\} \\
=\psi\left(w_{1}\right)=\psi\left(w_{2}\right)=\sup _{T}\left\{\theta\left(w_{2}^{\prime}\right): 0(x) \in A(x) \text { for } x \in Y\right\} .
\end{aligned}
$$


But $\eta\left(w_{1}^{\prime}\right)$ is isolated in $S$ by $T$. Hence there exists $\theta_{0}: X \rightarrow S$ such $\theta_{0}(x) \in A(x)=$ $\eta \phi^{-1} \phi(x)$ for all $x \in X$ and

$$
\eta\left(w_{1}^{\prime}\right)=\theta_{0}\left(w_{2}^{\prime}\right)
$$

Thus there exists $x^{\prime} \in \phi^{-1} \theta(x)$ such that $\eta\left(x^{\prime}\right)=\theta_{0}(x)$. Define $\theta^{\prime}$ by $\theta^{\prime}(x)=x^{\prime}$. We then have

$$
\eta \theta^{\prime}(x)=\eta\left(x^{\prime}\right)=\theta_{0}(x)
$$

whence $\eta \theta^{\prime}=\theta_{0}$. Further $\phi \theta^{\prime}(x)=\phi\left(x^{\prime}\right)=\phi(x)$ whence $\phi \theta^{\prime}=\phi$. Thus if we define $w_{2}^{\prime \prime}=\theta^{\prime}\left(w_{2}^{\prime}\right)$, then

and

$$
w_{2}^{\prime} \geqslant w_{2}^{\prime \prime}
$$

$$
\begin{aligned}
\left(\phi\left(w_{1}^{\prime}\right), \phi\left(w_{2}^{\prime \prime}\right)\right) & =\left(\phi\left(w_{1}^{\prime}\right), \phi \theta^{\prime}\left(w_{2}^{\prime}\right)\right) \\
& =\left(\phi\left(w_{1}^{\prime}\right), \phi\left(w_{2}^{\prime}\right)\right)=\left(w_{1}, w_{2}\right),
\end{aligned}
$$

whence

$$
\left(w_{1}^{\prime}, w_{2}^{\prime \prime}\right) \geqslant\left(w_{1}, w_{2}\right)
$$

Further

$$
\eta\left(w_{2}^{\prime \prime}\right)=\eta \theta^{\prime}\left(w_{2}^{\prime}\right)=\theta_{0}\left(w_{2}^{\prime}\right)=\eta\left(w_{1}^{\prime}\right)
$$

and the lemma is proved.

\section{(4.10) Conventions and Definitions}

(4.10.1) Henceforth, we shall assume that $X$ is a countably infinite set $X=$ $\left\{x_{0}, x_{1}, x_{2}, \ldots\right\}$, unless otherwise indicated.

(4.10.2) If $n$ is a cardinal, we define

$$
X_{n}=\left\{x_{i} \in X: i<n\right\}
$$

Hence $X_{n}=X$, if and only if $n=\mathfrak{\aleph}_{0}$, and for finite $n,\left|X_{n}\right|=n$.

(4.10.3) If $(\Omega, S)$ is an algebra free over itself with generating set $G$, we shall suppose that $G$ is well-ordered; $G=\left\{g_{0}, g_{1}, g_{2}, \ldots\right\}$. This is an inessential notation convenience.

(4.10.4) If $G$ is as in (4.10.3), and $|G|=n$, the homomorphism of $\left\langle\Omega, X_{n}\right\rangle$ into $\left(\Omega, S\right.$ ) given by $\eta\left(x_{i}\right)=g_{i}, i<n$ (or $i<\aleph_{0}$ ), will be called the natural homomorphism of $\left\langle\Omega, X_{n}\right\rangle$ into $(\Omega, G)$. Observe that $\eta$ is $1-1$ on $X_{n}, n \leqslant \aleph_{0}$.

(4.10.5) If $(\Omega, S)$ is free over itself and $\eta$ is the natural homomorphism of $\langle\Omega, X\rangle$ into $(\Omega, S)$, then for $\left(w_{1}, w_{2}\right) \in\left\langle\Omega, X_{n}\right\rangle^{2}$ we have $\left(w_{1}, w_{2}\right) \in \operatorname{Id}\left(S, X_{n}\right)$ if and only if $\eta\left(w_{1}\right)=\eta\left(w_{2}\right)$. For, if $\theta: X_{n} \rightarrow S$, then $\theta=\lambda \eta$, where $\lambda$ is a homomorphism of $(\Omega, S)$ onto itself. Hence $\eta\left(w_{1}\right)=\eta\left(w_{2}\right)$ implies that $\theta\left(w_{1}\right)=\theta\left(w_{2}\right)$.

(4.10.6) If $W \subseteq\langle\Omega, X\rangle^{2}$ then [W] will denote the smallest fully invariant congruence on $\langle\Omega, X\rangle$ which contains $W$. 
(4.10.7) If $W \subseteq\left\langle\Omega, X_{n}\right\rangle^{2}$, then $[W]_{n}$ denotes the smallest fully invariant congruence on $\left\langle\Omega, X_{n}\right\rangle$ containing $W$.

(4.10.8) It is easy to see that for $W \subseteq\left\langle\Omega, X_{n}\right\rangle^{2}$,

$$
[W] \subseteq \operatorname{Id}(S, X)
$$

if and only if

$$
[W]_{n} \subseteq \operatorname{Id}\left(S, X_{n}\right)
$$

(4.10.9) If $(\Omega, S)$ is free over itself on a finite number $n$ of generators it is possible that $[W]_{n}=\operatorname{Id}\left(S, X_{n}\right)$, but $[W] \subset \operatorname{Id}(S, X)$. For example, let $(\cdot, S)$ be the groupoid with generator $a$ satisfying $a^{2} \equiv a$. Then, in fact $S=\{a\}$, and $S$ is obviously free over itself. Clearly $\left(x, x^{2}\right)$ generates $\operatorname{Id}\left(S, X_{1}\right)$ but not $\operatorname{Id}(S, X)$. (A generator for $\operatorname{Id}(S, X)$ is $(x, y)$.) Similar, but less trivial examples may be given.

(4.11) LEMMA. Let $(\Omega, S)$ be a partially ordered algebra free over itself with $n$ generators, and let $(\Omega, T)$ be a suprema preserving superior completion of $(\Omega, S)$. Let $\left(w_{1}, w_{2}\right) \in \operatorname{Id}\left(T, X_{n}\right)$ and suppose there exists a linear pair $\left(w_{1}^{\prime}, w_{2}^{\prime}\right) \in\left\langle\Omega, X_{n}\right\rangle^{2}$ such that $\left(w_{1}^{\prime}, w_{2}^{\prime}\right) \geqslant\left(w_{1}, w_{2}\right)$ and $\eta\left(w_{1}^{\prime}\right)$ is isolated in $S$ by $T$, where $\eta$ is the natural mapping of $\left\langle\Omega, X_{n}\right\rangle$ into $(\Omega, S)$. Then there exists $\left(w_{1}^{\prime}, w_{2}^{\prime \prime}\right) \geqslant\left(w_{1}, w_{2}\right)$ such that $\left(w_{1}^{\prime}, w_{2}^{\prime \prime}\right) \in \operatorname{Id}\left(S, X_{n}\right)$.

Proof. Since $\left(w_{1}^{\prime}, w_{2}^{\prime}\right) \geqslant\left(w_{1}, w_{2}\right)$ there exists a mapping $\phi: X \rightarrow X$ such that $\phi\left(w_{i}^{\prime}\right)=w_{i}, i=1,2$. As in (4.9) with $X_{n}$ replacing $X$ define $Y=\Gamma\left(w_{1}^{\prime}\right) \cup \Gamma\left(w_{2}^{\prime}\right)$. Define $\psi: X_{n} \rightarrow T$ as follows:

(1) If $z \in \phi(Y)$ with $z=\phi(x)$ then

$$
\psi(z)=\sup _{T} \eta\left(\phi^{-1} \phi(x)\right) .
$$

(2) If $z \in X_{n} \backslash \phi(Y)$, define

$$
\psi(z)=\eta(z)
$$

Since $\left(w_{1}, w_{2}\right) \in \operatorname{Id}\left(T, X_{n}\right)$ we have $\psi\left(w_{1}\right)=\psi\left(w_{2}\right)$. Hence by (4.9) there exists $w_{2}^{\prime \prime} \epsilon$ $\epsilon\left\langle\Omega, X_{n}\right\rangle$ with $\left(w_{1}, w^{\prime \prime}\right) \geqslant\left(w_{1}^{\prime}, w_{2}^{\prime \prime}\right)$ and $\eta\left(w_{1}^{\prime}\right)=\eta\left(w_{2}^{\prime \prime}\right)$. But since $\eta$ is the natural map, $\left(w_{1}^{\prime}, w_{2}^{\prime \prime}\right) \in \operatorname{Id}\left(S, X_{n}\right)(\mathrm{cf} .(4.10 .4))$.

\section{(4.12) Remark}

It follows from (4.8.4) that the isolation hypothesis in (4.11) is automatically fulfilled when $T=\mathscr{S}(S)$ and $S$ is trivially ordered.

(4.13) THEOREM. Let $(\Omega, S)$ be a partially ordered algebra, free over itself on an infinity of generators. Let $(\Omega, T)$ be a suprema preserving superior completion, and suppose for each linear $v \in\langle\Omega, X\rangle, \eta(v)$ is isolated in $S$ by $T$, where $\eta$ is the natural 
mapping of $\langle\Omega, X\rangle$ into $(\Omega, S)$. Then if $\left(w_{1}, w_{2}\right) \in \operatorname{Id}(T, X)$ there exists a linear pair $\left(v_{1}, v_{2}\right)$ such that $\left(w_{1}, w_{2}\right) \leqslant\left(v_{1}, v_{2}\right)$ and $\left(v_{1}, v_{2}\right) \in \operatorname{Id}(S, X)$.

Proof. Since $X$ is infinite, there exists a linear pair $\left(w_{1}^{\prime}, w_{2}^{\prime}\right)$ with $\left(w_{1}, w_{2}\right) \leqslant\left(w_{1}^{\prime}, w_{2}^{\prime}\right)$ (cf. 2.7), and also $X_{n}=X$. Since $\eta\left(w_{1}^{\prime}\right)$ is isolated, it follows from (4.11) that there exists $\left(w_{1}^{\prime}, w_{2}^{\prime}\right) \in \operatorname{Id}(S, X)$ with $\left(w_{1}^{\prime}, w_{2}^{\prime \prime}\right) \geqslant\left(w_{1}, w_{2}\right)$. Since $\left(w_{2}^{\prime \prime}, w_{1}^{\prime}\right) \in \operatorname{Id}(S, X)$, we may repeat this argument to obtain $\left(v_{1}, v_{2}\right)$ such that $v_{2}$ is linear, $\left(v_{1}, v_{2}\right) \geqslant\left(w_{1}^{\prime}, w_{2}^{\prime \prime}\right)$ and $\left(v_{1}, v_{2}\right) \in \operatorname{Id}(S, X)$. But since $v_{2} \geqslant w_{1}^{\prime}$, and $w_{1}^{\prime}$ is linear it follows that $v_{2}$ is also linear, and the theorem is proved.

(4.14) THEOREM. Let $(\Omega, S)$ be a partially ordered algebra, free over itself on $n$ generators. Let $(\Omega, T)$ be a suprema preserving superior completion. Suppose there exists a subset $\mathscr{W} \subseteq\left\langle\Omega, X_{n}\right\rangle^{2}$ such that $[W]=\operatorname{Id}(S, X)$ and which satisfies:

(4.14.1) for each $\left(v_{1}, v_{2}\right) \in W$ there exists a chain

such that

$$
\left(v_{1}, v_{2}\right) \leqslant\left(w_{1}, w_{2}\right) \leqslant\left(w_{1}^{\prime}, w_{2}^{\prime}\right)
$$

(1) $\left(w_{1}, w_{2}\right)$ is maximal in $\operatorname{Id}\left(S, X_{n}\right)$,

(2) $\left(w_{1}^{\prime}, w_{2}^{\prime}\right) \in\left\langle\Omega, X_{n}\right\rangle$,

(3) $\left(w_{1}^{\prime}, w_{2}^{\prime}\right)$ is linear,

and

(4) both $\eta\left(w_{1}^{\prime}\right)$ and $\eta\left(w_{2}^{\prime}\right)$ are isolated in $S$ by $T$, where $\eta$ is the natural mapping of $\left\langle\Omega, X_{n}\right\rangle$ into $(\Omega, S)$.

Then $\operatorname{Id}(T, X)=\operatorname{Id}(S, X)$ if and only if there exists a set $Z$ of linear pairs in $\left\langle\Omega, X_{n}\right\rangle^{2}$ such that $[Z]=\operatorname{Id}(S, X)$.

Proof. Clearly $\operatorname{Id}(T, X) \subseteq \operatorname{Id}(S, X)$ since $(\Omega, S)$ is isomorphically embedded in $(\Omega, T)$. Suppose $\operatorname{Id}(S, X)$ contains a set $Z$ of linear generators with $Z \subseteq\langle\Omega, X\rangle^{2}$. Then by [1] Theorem (5.30) each $\left(w_{1}, w_{2}\right) \in Z$ is an identity on $(\Omega, T)$, whence $\operatorname{Id}(S, X)$ $=[Z] \subseteq \operatorname{Id}(T, X)$ and $\operatorname{Id}(T, X)=\operatorname{Id}(S, X)$ follows. This proves the theorem in one direction.

Conversely, suppose $\operatorname{Id}(T, X)=\operatorname{Id}(S, X)$. For each $\left(v_{1}, v_{2}\right) \in W$ choose a chain

$$
\left(v_{1}, v_{2}\right) \leqslant\left(w_{1}, w_{2}\right) \leqslant\left(w_{1}^{\prime}, w_{2}^{\prime}\right)
$$

satisfying hypotheses (1)-(4) and let $Z$ consist of all pairs $\left(w_{1}, w_{2}\right)$ which belong to one of the chosen chains. We shall show that $\left(w_{1}, w_{2}\right)$ in $Z$ is linear. Observe that $\left(w_{1}, w_{2}\right) \in \operatorname{Id}\left(S, X_{n}\right)$. Since $\eta\left(w_{1}^{\prime}\right)$ is isolated, by (4.11) there exists $w_{2}^{\prime \prime}$ such that $\left(w_{1}^{\prime}\right.$, $\left.w_{2}^{\prime \prime}\right) \in \operatorname{Id}\left(S, X_{n}\right)$ and $\left(w_{1}^{\prime}, w_{2}^{\prime \prime}\right) \geqslant\left(w_{1}, w_{2}\right)$. But by the maximality of $\left(w_{1}, w_{2}\right)$ in $\operatorname{Id}\left(S, X_{n}\right)$, $\left(w_{1}^{\prime}, w_{2}^{\prime \prime}\right) \sim\left(w_{1}, w_{2}\right)$ whence $w_{1} \geqslant w_{1}^{\prime}$. Clearly (cf. (2.8)), $w_{1}$ is also linear. Since $\left(w_{1}, w_{2}\right)$ is maximal in $\operatorname{Id}\left(S, X_{n}\right)$, so is $\left(w_{2}, w_{1}\right)$. Further $\left(w_{2}^{\prime}, w_{1}^{\prime}\right) \geqslant\left(w_{2}, w_{1}\right)$ and $\eta\left(w_{2}^{\prime}\right)$ is 
isolated. A similar argument now shows that $w_{2}$ is linear. Hence $\left(w_{1}, w_{2}\right)$ is a linear pair. We have shown that $Z$ consists of linear pairs. Since $W \subseteq[Z]$, and $Z \subseteq \operatorname{Id}(S, X)$ we have

$$
[Z]=[W]=\operatorname{Id}(S, X) .
$$

(4.15) THEOREM. Let $(\Omega, S)$ be a partially ordered algebra, free over itself on $n$ generators, and let $(\Omega, T)$ be a suprema preserving superior extension. Suppose there exists a subset $V$ of $\langle\Omega, X\rangle^{2}$ such that $[V]=\operatorname{Id}(S, X)$, and which satisfies:

(4.15.1) For each $\left(u_{1}, u_{2}\right) \in V$, we have

$$
\mu\left(u_{1}\right)+\mu\left(u_{2}\right) \leqslant n .
$$

(4.15.2) Suppose also that for each linear pair $\left(w_{1}^{\prime}, w_{2}^{\prime}\right) \in\left\langle\Omega, X_{n}\right\rangle^{2}$ there exists $\left(w_{1}^{\prime \prime}, w_{2}^{\prime \prime}\right) \in\left\langle\Omega, X_{n}\right\rangle^{2}$, such that $\left(w_{1}^{\prime \prime}, w_{2}^{\prime \prime}\right) \geqslant\left(w_{1}^{\prime}, w_{2}^{\prime}\right)$ and both $\eta\left(w_{1}^{\prime \prime}\right)$ and $\eta\left(w_{2}^{\prime \prime}\right)$ are isolated. Then $\operatorname{Id}(T, X)=\operatorname{Id}(S, X)$ if and only if there exists a set of linear pairs $Z$ in $\left\langle\Omega, X_{n}\right\rangle^{2}$ such that $\operatorname{Id}(S, X)=[Z]$.

Proof. Let $\left(u_{1}, u_{2}\right) \in V$. Since

$$
\gamma\left(u_{1}, u_{2}\right) \leqslant \mu\left(u_{1}\right)+\mu\left(u_{2}\right) \leqslant n,
$$

there exists a permutation $\pi$ on $X$ such that $\pi\left(\Gamma\left(u_{1}, u_{2}\right)\right) \subseteq X_{n}$. Let $v_{i}=\pi\left(u_{i}\right), i=1,2$. Then $\left(v_{1}, v_{2}\right) \in \operatorname{Id}\left(S, X_{n}\right)$, and $\left(v_{1}, v_{2}\right) \sim\left(u_{1}, u_{2}\right)$. Hence we can find a set of generators $W$ for $\operatorname{Id}(S, X)$ such that

$$
W \subseteq \operatorname{Id}\left(S, X_{n}\right) \subset\left\langle\Omega, X_{n}\right\rangle^{2}
$$

and

$$
\mu\left(v_{1}\right)+\mu\left(v_{2}\right) \leqslant n,
$$

for each $\left(v_{1}, v_{2}\right) \in W$. By (2.6), there exists $\left(w_{1}, w_{2}\right) \in \operatorname{Id}\left(S, X_{n}\right)$ such that $\left(w_{1}, w_{2}\right)$ is maximal in $\operatorname{Id}\left(S, X_{n}\right)$ and $\left(w_{1}, w_{2}\right) \geqslant\left(v_{1}, v_{2}\right)$. Since $w_{i} \geqslant v_{i}, i=1,2$, we have

(cf. (2.2.6)) whence

$$
\mu\left(w_{i}\right)=\mu\left(v_{i}\right)
$$

$$
\mu\left(w_{1}, w_{2}\right) \leqslant \mu\left(w_{1}\right)+\mu\left(w_{2}\right) \leqslant \mu\left(v_{1}\right)+\mu\left(v_{2}\right) \leqslant n .
$$

Thus by (2.7) there exists a linear pair $\left(w_{1}^{\prime}, w_{2}^{\prime}\right) \in\langle\Omega, X\rangle^{2}$ with $\left(w_{1}^{\prime}, w_{2}^{\prime}\right) \geqslant\left(w_{1}, w_{2}\right)$. By assumption, we can then find a linear pair $\left(w_{1}^{\prime \prime}, w_{2}^{\prime \prime}\right) \in\left\langle\Omega, X_{n}\right\rangle^{2}$, such that $\left(w_{1}^{\prime \prime}, w_{2}^{\prime \prime}\right) \geqslant$ $\geqslant\left(w_{1}^{\prime}, w_{2}^{\prime}\right)$ and $\eta\left(w_{1}^{\prime \prime}\right)$ and $\eta\left(w_{2}^{\prime \prime}\right)$ are isolated in $S$ by $T$. Thus Theorem (4.14) applies and the conclusion follows.

(4.16) THEOREM. Let $(\Omega, S)$ be a partially ordered algebra, free over itself on $n$ generators, and let $(\Omega, T)$ be a suprema preserving superior extension. Suppose there exists a subset $V$ of $\langle\Omega, X\rangle^{2}$ such that $[V]=\operatorname{Id}(S, X)$, and which satisfies: 
(4.16.1) Each pair $\left(u_{1}, u_{2}\right)$ in $V$ is maximal in $\operatorname{Id}(S, X)$ and satisfies $\mu\left(u_{1}, u_{2}\right) \leqslant n$.

(4.16.2) Suppose that for each linear pair $\left(w_{1}^{\prime}, w_{2}^{\prime}\right) \in\left\langle\Omega, X_{n}\right\rangle^{2}$ there exists $\left(w_{1}^{\prime \prime}, w_{2}^{\prime \prime}\right) \in$ $\in\left\langle\Omega, X_{n}\right\rangle^{2}$ such that $\left(w_{1}^{\prime \prime}, w_{2}^{\prime \prime}\right) \geqslant\left(w_{1}^{\prime}, w_{2}^{\prime}\right)$ and both $\eta\left(w_{1}^{\prime \prime}\right)$ and $\eta\left(w_{2}^{\prime \prime}\right)$ are isolated.

Then $\operatorname{Id}(T, X)=\operatorname{Id}(S, X)$ if and only if there exists a set of linear pairs $Z$ in $\langle\Omega, X\rangle^{2}$ such that $\operatorname{Id}(S, X)=[Z]$.

Proof. Similar to (4.15).

We may again observe that for trivially ordered $S$ with $T=\mathscr{P}(S)$ the hypotheses involving isolation are trivially satisfied. We shall state an important special case of (4.15).

(4.17) THEOREM. Let $(\Omega, S)$ be a trivially ordered algebra, free over itself with an infinite set of generators. Then $\operatorname{Id}(\mathscr{S}(S), X)=\operatorname{Id}(S, X)$ if and only if there exists a set $Z$ of linear pairs such that $[Z]=\operatorname{Id}(S, X)$.

Let $W$ be contained in $\langle\Omega, X\rangle^{2}$ where $X$ is countably infinite. The equational class (variety) $\mathscr{K}(W)$ defined by $W$ consists of all algebras $(\Omega, S)$ with $\operatorname{Id}(S, X) \supseteq W$. Let $\mathscr{K}=\mathscr{K}(W)$ be an equational class. Then $\operatorname{Id}(\mathscr{K}, X)$ is defined to be the set pairs $\left(w_{1}, w_{2}\right)$ such that $w_{1} \equiv w_{2}$ holds on $S$, for each $S \in \mathscr{K}$. We include the following, which must be known, but we cannot find it in the form needed.

(4.18) LEMMA. Let $X$ be infinite, and let $W \subseteq\langle\Omega, X\rangle^{2}$. Let $\mathscr{K}$ be an equational class. Then $\mathscr{K}=\mathscr{K}(W)$ if and only if $[W]=\operatorname{Id}(\mathscr{K}, X)$.

Proof. Let $\mathscr{K}=\mathscr{K}(W)$. Let $(\Omega, S) \in \mathscr{K}(W)$. Then, since $\operatorname{Id}(S, X)$ is a fully invariant congruence containing $W$, we have

$$
[W] \subseteq \operatorname{Id}(S, X)
$$

But the algebra $\left(\Omega, S_{1}\right)=\langle\Omega, X\rangle /[W] \in \mathscr{K}(W)$ and by $(4.3)$,

$$
\operatorname{Id}\left(S_{1}, X\right)=[W] \supseteq W .
$$

Thus

$$
\begin{aligned}
{[W] } & =\bigcap\{\operatorname{Id}(S, X): S \in \mathscr{K}(W)\} \\
& =\operatorname{Id}(\mathscr{H}(W), X)=\operatorname{Id}(\mathscr{K}, X) .
\end{aligned}
$$

Conversely, let $[W]=\operatorname{Id}(\mathscr{K}, X)$. Then $S \in \mathscr{K}(W)$ if and only if $\operatorname{Id}(S, X) \supseteq[W]$ $=\operatorname{Id}(\mathscr{K}, X)$. But $\operatorname{Id}(S, X) \supseteq \operatorname{Id}(\mathscr{K}, X)$ if and only if $S \in \mathscr{K}$. Thus $\mathscr{K}=\mathscr{K}(W)$.

We define $(\Omega, \mathscr{C}(S))$, the complex algebra to be the induced algebra on the set of all non-empty subsets of $S$, i.e. if $S$ is trivially ordered $\mathscr{C}(S)=\mathscr{I}(S)$. We define $\mathscr{C}(\mathscr{K})$ to be the class of all $\mathscr{C}(S)$ with $S \in \mathscr{K}$.

(4.19) THEOREM. Let $\mathscr{K}$ be an equational class. Then $\mathscr{C}(\mathscr{K}) \subseteq \mathscr{K}$ if and only if there exists a subset $W$ of $\langle\Omega, X\rangle^{2}$ consisting of linear pairs such that $\mathscr{K}=\mathscr{K}(W)$. 
Proof. Let $\mathscr{K}=\mathscr{K}(W)$, where $W$ consists of linear pairs and $S \in \mathscr{K}$. Let $\left(w_{1}, w_{2}\right) \in W$. Since $w_{1} \equiv w_{2}$ holds on $S$ and $\left(w_{1}, w_{2}\right)$ is linear it follows by [1] Theorem 5.30 that $w_{1} \equiv w_{2}$ holds on $\mathscr{C}(S)$. Hence $\mathscr{C}(S) \in \mathscr{K}$. Thus $\mathscr{C}(\mathscr{K}) \subseteq \mathscr{K}$.

Conversely, let $\mathscr{C}(\mathscr{K}) \subseteq \mathscr{K}$. Consider

$$
(\Omega, S)=\langle\Omega, X\rangle / \mathrm{Id}(\mathscr{K}, X)
$$

If $(\Omega, S)$ has one element, then $\operatorname{Id}(\mathscr{K}, X)=\langle\Omega, X\rangle^{2}$ and hence $\operatorname{Id}(\mathscr{K}, X)=[W]$, where $W=\left\{(x, y) \in X^{2}\right\}$, a set of linear pairs. If $|(\Omega, S)|>1$, then $(\Omega, S)$ is free over itself with an infinity of generators, by Theorem (4.5). Hence, by (4.17) there exists a set $W$ of linear pairs with $[W]=\operatorname{Id}(\mathscr{K}, X)$. By Lemma $(4.18) \mathscr{K}=\mathscr{K}(W)$, and the theorem is proved.

\section{(4.20) Examples}

We shall show by means of two examples, one trivially ordered and one linearly ordered, that we cannot omit the hypothesis in $(4.11)$ that $\left(w_{1}^{\prime}, w_{2}^{\prime}\right)$ be in $\left(\Omega, X_{n}\right)^{2}$.

(4.20.1) Let $S$ be the positive integers under two operations: $a+b=$ the ordinary sum and $a \wedge b=\max (a, b)$. Then $S$ is free over itself with generating set $\{1\}$, and $(+, \wedge, S)$ satisfies the identity $x \wedge x \equiv x$. Let $S$ be trivially ordered. Then $x \wedge x \equiv x$ also holds on $(t, \wedge, \mathscr{S}(S))$. The linear pair $(x \wedge y, x)$ is above $(x \wedge x, x)$. And up to permutations of symbols, the only pairs $\left(x \wedge y, w_{2}^{\prime \prime}\right) \geqslant(x \wedge x, x)$ are $(x \wedge y, x)$, $(x \wedge y, y),(x \wedge y, z)$ and none of them correspond to an identity on $(+, \wedge, S)$. Observe that $n=1$ and $(x \wedge y, x) \notin\left\langle\Omega, X_{1}\right\rangle^{2}$, if $y \neq x$.

(4.20.2) Similar remarks hold about $x \wedge x=x$ if $S$ is ordered in reverse fashion from the usual (e.g., $2>3$ ). Note that $\eta\left(x_{0}\right)=1$ is isolated in $S$ by $\mathscr{S}(S)$ in this case.

\section{(4.21) Examples}

We now give two examples to show that the isolation hypothesis (4.15.2) can be satisfied for non-trivially ordered $S$, with $(\Omega, S)$ free over itself on an infinity of generators.

(4.21.1) Let $S$ be the multiplicative semigroup of all integers $a, a \geqslant 2$, and order $S$ thus: $a \leqslant b$ if and only if

(1) $b \mid a$

and

(2) for some integer $n, a \mid b^{n}$.

(or, equivalently, (1) and $(2)^{\prime}: a$ and $b$ have the same prime factors). Then, $S$ is free over itself, generated by the set of primes $=\left\{p_{0}, p_{1}, \ldots\right\}$ and the maximal elements of $S$ are products of distinct primes. Since for each $x \in X, \eta(x)$ is a prime, it follows that 
any linear word $w^{\prime}$ in $\langle\Omega, X\rangle$ maps onto a maximal element of $S$, and hence $\eta\left(w^{\prime}\right)$ is isolated in $\mathscr{S}(S)$. In this case $\mathrm{Id}(\cdot, S)$ has a generating set $\{(x y) z=x(y z), x y=y x\}$ and hence

$$
\operatorname{Id}(\Omega, S)=\operatorname{Id}(\Omega, \mathscr{S}(S)) .
$$

(4.21.2) Let $S$ be the set of all cube free integers $a, a \geqslant 2$. If $a=\prod p_{i}^{\alpha_{i}}, b=\prod p_{i}^{\beta_{i}}$, $0 \leqslant \alpha_{i} \leqslant 2,0 \leqslant \beta_{i} \leqslant 2$, define $c=a$ ob by $c=\prod p_{i}^{\gamma_{i}}$, where $\gamma_{i}=\min \left(\alpha_{i}+\beta_{i}, 2\right)$. It can be shown that $S$ is free over itself with generating set the primes $\left\{p_{0}, p_{1}, \ldots\right\}$. If we order $S$ by (1) and (2) of (4.21.1), then it again follows that $\eta\left(w^{\prime}\right)$ is isolated by $S$ in $\mathscr{S}(S)$, for any linear $w^{\prime} \in(\Omega, X)$. In this case, however, $(x \circ x) \circ x=x \circ x$ holds on $(\circ, S)$, but not on $(\circ, \mathscr{S}(S))$. To see this, let $I$ be the initial segment generated by $\{2,3,5\}$.)Then $30 \notin I \circ I$, but $30 \in(I \circ I) \circ I$. Hence by (4.15), there exists no linear set of generators for $\operatorname{Id}(S, X)$. We observe that $\operatorname{Id}(S, X)=\mathscr{R}$, where we define

$$
\mathscr{R}=\left\{\left(w_{1}, w_{2}\right): \min \left(2, \mu_{x}\left(w_{1}\right)\right)=\min \left(2, \mu_{x}\left(w_{2}\right)\right) \text { for all } x \in X\right\} .
$$

For future reference, we point out that if we adjoin 1 as an incomparable element of $S$ and also as a nullary operation, then all above remarks go through.

\section{(4.22) Example}

We give an example of a trivially ordered semigroup $S$ satisfying all hypotheses of (4.15), and such that $\operatorname{Id}(\mathscr{S}(S), X) \neq \operatorname{Id}(S, X)$. Let $S$ be the square-free positive integers $a(a \geqslant 2)$ under the composition $a \wedge b=1$.c.m. $(a, b)$. Then $S$ is free and is generated by the primes. If $S$ is trivially ordered, the hypotheses (4.15.2) and (4.15.3) hold. Define $\mathscr{2}$ by:

$$
\mathscr{Q}=\left\{\left(w_{1}, w_{2}\right) \in\langle\Omega, X\rangle^{2}: \Gamma\left(w_{1}\right)=\Gamma\left(w_{2}\right)\right\} .
$$

It follows from the identities $x \wedge x \equiv x,(x \wedge y) \wedge z \equiv x \wedge(y \wedge z)$ and $x \wedge y \equiv y \wedge x$ that $w_{1} \equiv w_{2}$ holds on $(\wedge, S)$ if and only if $\left(w_{1}, w_{2}\right) \in \mathscr{Q}$, thus $\mathscr{Q}=\operatorname{Id}(S, X)$. If $A=\{2,3\} \subseteq S$, then $\{2,3\} \wedge\{2,3\}=\{2,3,6\}$, whence $x \wedge x \equiv x$ does not hold on $(\wedge, \mathscr{S}(S))$. Thus by Theorem (4.15), $\operatorname{Id}(S, X)=\mathscr{Q}$ does not have a set of linear generators.

\section{(4.23) Examples}

We now show that none of the hypotheses of Theorem (4.15), viz., that $S$ be free over itself, (4.15.1), or (4.15.2) can be omitted.

(4.23.1) Let $S$ be the positive integers with $a \wedge b=\max (a, b)$. The only generating set for $S$ is $S$ itself, and $S$ is not free over itself. (To see this, observe that any mapping with $1 \rightarrow 2$ and $2 \rightarrow 1$ cannot be extended to a homomorphism.) If $S$ is trivially ordered, (4.15.1) and (4.15.2) are satisfied. We note that $x \wedge x \equiv x,(x \wedge y) \wedge z \equiv x \wedge(y \wedge z)$, $x \wedge y \equiv y \wedge x$ all hold in both $S$ and $\mathscr{S}(S)$. It follows that $\operatorname{Id}(S, X)=\operatorname{Id}(\mathscr{S}(S), X)=\mathscr{Q}$, 
where $\mathscr{Q}$ is given by (4.22.1). But as was shown in (4.22), there is no set of linear pairs generating $\mathscr{Z}$.

(4.23.2) We next show that hypothesis (4.15.1) cannot be omitted from Theorem (4.15). Let $S=\{1, a, 0\}$ have a multiplication where 1 and 0 are the identity and zero elements and $a^{2}=0$. Also let 1 be a nullary operation. Thus $\Omega=\{\cdot, 1\}$, and $S$ is free over itself with generator $a$. It can be proved that $\operatorname{Id}(\Omega, S)=\mathscr{R}$, where $\mathscr{R}$ is defined by $(4.21 .2 .1)$.

Let $S$ be trivially ordered. It is straightforward to check that $\operatorname{Id}(S, X)=\operatorname{Id}(\mathscr{S}(S)), X$. Since we have already shown $\operatorname{Id}(S, X)$ is not generated by linear pairs we see that hypothesis (4.15.1) is essential.

(4.23.3) We may generalize the example of (4.23.2). For each positive integer $n$, we may construct the trivially ordered algebra $S=\left\{1, a, a^{2}, \ldots, a^{n-1}, 0\right\}$ with an associative, commutative multiplication and a nullary operation 1 . The algebra is free over itself and satisfies $x^{n+1} \equiv x^{n}$ where $x^{n}$ may be associated in any fashion. As in (4.23.2), we prove that $\operatorname{Id}(S, X)=\operatorname{Id}(\mathscr{P}(S), X)$, but that $\operatorname{Id}(S, X)$ is not generated by linear pairs.

(4.23.4) Let $S$ be the semigroup of (4.22) and order $S$ by $a \leqslant b$ if and only if $b \mid a$. Then $S$ is free over itself with an infinity of generators, whence also (4.15.2) must hold. In Example (5.4) we shall show that $\operatorname{Id}(S, X)=\operatorname{Id}(\mathscr{P}(S), X)$. We have already seen in (4.22) that $\operatorname{Id}(S, X)$ has no set of linear generators.

\section{(4.25) More examples and remarks}

(4.24.1) Let $S$ be a trivially ordered algebra free over itself on $n$ generators, where $n$ is finite. In view of (4.23.2), it is tempting to conjecture that if $\left(w_{1}, w_{2}\right)$ is a maximal element of $\operatorname{Id}\left(S, X_{n}\right)$, then also $\left(w_{1}, w_{2}\right) \in \operatorname{Id}\left(\mathscr{P}(S), X_{n}\right)$. However, this is false, as shown by the following example:

Let $S$ be the positive integers with two operations + and $\circ$ where + is ordinary addition and for $a, b \in S$ define

$$
a \circ b= \begin{cases}a & \text { if } a=b \\ a+b & \text { otherwise }\end{cases}
$$

Then $S$ is free over itself with generator 1 . Consider $S$ under the trivial order. Then $(x \circ x, x)$ is maximal in $\operatorname{Id}\left(S, X_{1}\right)$, but $(x \circ x, x) \notin \operatorname{Id}\left(\mathscr{P}(S), X_{1}\right)$.

(4.24.2) A final example shows that if $S=\left\langle\Omega, X_{n}\right\rangle /[V]_{n}$, it may happen that $[V] \neq \operatorname{Id}(S, X)$. For example, let $S$ be a groupoid, free over itself on one generator $a$, satisfying

$$
\begin{aligned}
x\left(x^{2}\right) & =x^{2} \quad \text { and } \quad\left(x^{2}\right) x=x^{2}, \text { i.e. } \\
V & =\left\{\left(x x^{2}, x^{2}\right),\left(x^{2} x, x^{2}\right)\right\} \quad \text { and } n=1 .
\end{aligned}
$$

Then $S \cong\left\{a, a^{2}\right\}$. Clearly $x(y z) \equiv x y$ holds on $(\circ, S)$ (so that $\left(x\left(x^{2}\right), x^{2}\right)$ can be replaced 
by a linear pair). However, the congruence $[V]$ does not contain $(x(y z), x y)$, since the semigroup of (4.21.2) satisfies $[V]$, but not $x y z \equiv x y$.

\section{§5. Local conditions}

We now find a local condition on the partial order which for each $w$ is equivalent to the condition $\psi(w)=\psi(w)$. This latter condition was the main criterion for the permanence of identities in the paper [1]. After proving the theorem we give an example to show that this condition is, in fact, not necessary for the permanence of identities under extension of the algebra to a given superior completion.

\section{(5.1) Definitions}

Let $w, w^{\prime} \in\langle\Omega, X\rangle$. Let $(\Omega, S)$ be a partially ordered algebra with suprema preserving superior completion $(\Omega, T)$.

(5.1.1.) We say $w$ covers $w^{\prime}$ in $S$ by $T$ if and only if there exists $\phi: X \rightarrow X$ with $\phi\left(w^{\prime}\right)=w$ and for each $\theta: X \rightarrow S$ we have

$$
\theta\left(w^{\prime}\right) \leqslant \sup _{T}\left\{\theta^{*}(w): \theta^{*} \phi(x) \in \theta \phi^{-1} \phi(x)\right\} .
$$

(5.1.2) Let $w^{\prime} \in\langle\Omega, X\rangle$. We say that $w^{\prime}$ is totally covered in $S$ by $T$ if every $w \in\langle\Omega, X\rangle$ with $w \leqslant w^{\prime}$ covers $w^{\prime}$ in $S$ by $T$.

(5.2) THEOREM. Let $(\Omega, S)$ be a partially ordered algebra with suprema preserving superior completion $(\Omega, T)$. Let $w$ be a word in $\langle\Omega, X\rangle$. Then for every $\psi: X \rightarrow T, \psi(w)=\psi(w)$ if and only if there exists a linear $w^{\prime} \in\langle\Omega, X\rangle$ such that $w$ covers $w^{\prime}$ in $S$ by $T$.

Proof. Suppose $\psi(w)=\psi(w)$, for all $\psi: X \rightarrow T$. By (2.8) there exists $w^{\prime} \geqslant w$ where $w^{\prime}$ is linear. Suppose $\phi\left(w^{\prime}\right)=w$. Let $\theta: X \rightarrow S$ be given. We define

$$
\psi(y)=\left\{\begin{array}{lll}
\sup _{T} \theta \phi^{-1}(y) & \text { for } & y \in \phi(X), \\
0(y) & \text { for } & y \in X \backslash \phi(X) .
\end{array}\right.
$$

Thus, for $x \in X, \theta(x) \in \theta \phi^{-1} \phi(x)$, whence $\theta(x) \leqslant \psi \phi(x)$. Hence

$$
\begin{aligned}
\theta\left(w^{\prime}\right) & \leqslant \psi \phi\left(w^{\prime}\right)=\psi(w)=\psi(w) \\
& =\sup _{T}\left\{\theta^{*}(w): \theta^{*}(w) \leqslant \psi(x)\right\} \\
& =\sup _{T}\left\{\theta^{*}(w): \theta^{*}(y) \in \theta \phi^{-1}(y), y \in \phi(x)\right\},
\end{aligned}
$$

where the last equality holds by (3.2), with $A(y)=\theta \phi^{-1}(y)$ for $y \in \phi(x)$, and the previous one from the definition of $\psi(w)$. Hence $w$ covers $w^{\prime}$ in $S$ by $T$.

Now conversely, assume $w$ covers $w^{\prime}$ in $S$ by $T$. Let $\phi: X \rightarrow X$ be a mapping 
satisfying (5.1.1.1). Then by Theorem (3.2), with $A(x)=\{s \leqslant \psi \phi(x)\}$, we have

$$
\psi(w)=\sup _{r}\left\{\theta\left(w^{\prime}\right): \theta(x) \leqslant \psi \phi(x)\right\}
$$

Hence, by (4.1.1),

$$
\begin{aligned}
\psi(w) & \leqslant \sup _{r}\left\{\sup _{T} \theta^{*}(w): \theta^{*} \phi(x) \in \theta \phi^{-1} \phi(x) \text { and } \theta(x) \leqslant \psi \phi(x)\right\} \\
& =\sup _{r}\left\{\theta^{*}(w): \theta^{*} \phi(x) \in \theta \phi^{-1} \phi(x) \text { and } \theta(x) \leqslant \psi \phi(x)\right\}
\end{aligned}
$$

But if $\theta^{*} \phi(x) \in \theta \phi^{-1} \phi(x)$ and $\theta(x) \leqslant \psi \phi(x)$ then $\theta^{*} \phi(x)=\theta(z)$, where $\phi(z)=\phi(x)$, whence

$$
\theta^{*} \phi(x)=\theta(z) \leqslant \psi \phi(z)=\psi \phi(x)
$$

Thus

$$
\begin{aligned}
\psi(w) & \leqslant \sup _{r}\left\{\theta^{*}(w): \theta^{*} \phi(x) \leqslant \psi \phi(x)\right\} \\
& =\sup _{r}\left\{\theta^{*}(w): \theta^{*}(y) \leqslant \psi(y), y \in \phi(X)\right\} \\
& =\psi(w)
\end{aligned}
$$

where the last equality holds by (3.3) if $y \in \phi(X)$. Hence $\psi(w) \leqslant \psi(w)$. But $\psi(w) \leqslant \psi(w)$ by $[1](5.27)$, whence $\psi(w)=\psi(w)$, and the theorem is proved.

(5.3) THEOREM. Let $(\Omega, S)$ be a partially ordered algebra, and let $(\Omega, T)$ be a suprema preserving superior completion. If every linear word in $\langle\Omega, X\rangle$ is totally covered in $S$ by $T$ then $\operatorname{Id}(T, X)=\operatorname{Id}(S, X)$.

Proof. Let $\left(w_{1}, w_{2}\right) \in \operatorname{Id}(S, X)$, and let $\psi: X \rightarrow T$. It follows from the definition of $\psi$ that $\psi\left(w_{1}\right)=\psi\left(w_{2}\right)$. Hence by Theorem (5.2), also $\psi\left(w_{1}\right)=\psi\left(w_{2}\right)$, whence $\left(w_{1}, w_{2}\right) \in \operatorname{Id}(T, X)$. We have proved $\operatorname{Id}(S, X) \subseteq \operatorname{Id}(T, X)$. Since $S$ is embedded in $T$, the converse inclusion is trivial and the theorem follows.

\section{(5.4) Remark and examples}

(5.4.1) If $(\Omega, S)$ is linearly ordered, then it is easy to see that every linear word in $\langle\Omega, X\rangle$ is totally covered in $S$ by every superior completion $T$. Hence Theorem (5.3) implies Theorem (6.2) of [1].

(5.4.2) We now give an example of an algebra $(\Omega, S)$ which is not linearly ordered, and every linear word in $\langle\Omega, X\rangle$ is totally covered in $S$ by every superior completion $T$.

Let $S$ be the groupoid free over itself on a countable infinity of generators $\left\{p_{0}, p_{1}, p_{2}, \ldots\right\}$, which satisfies the identities $(x y) z=x(y z), x y \equiv y x, x^{2}=x$. Thus for every $a \in S$ we have a canonical representation

$$
a=\prod p_{i}^{\varepsilon_{i}}, \varepsilon_{i}=1 \text { or } \varepsilon_{i}=0 \text {, }
$$

where only finitely many $\varepsilon_{i} \neq 0$. (A natural model is given by the square-free in tegers 
$a(a \geqslant 2)$ with $a b=$ l.c.m. $(a, b)$, (cf. (4.22)), where we write $a \wedge b$.$) We partially order$ $S$ by

$$
a \leqslant b \quad \text { if and only if } b \text { divides } a \text {. }
$$

Let $w^{\prime}$ be linear and suppose $w \leqslant w^{\prime}$. There exists $a \phi: X \rightarrow X$ such that $\phi\left(w^{\prime}\right)=w$ and such that for $x \notin \Gamma\left(w^{\prime}\right)$ we have $\phi(x) \notin \Gamma(w)$. Then if $y \in \Gamma(w)$,

$$
\phi^{-1}(y) \subseteq \Gamma\left(w^{\prime}\right)
$$

Suppose $\theta^{*}(y) \in \theta \phi^{-1}(y)$. Then for some $x \in \Gamma\left(w^{\prime}\right), \theta^{*}(y)=\theta(x)$. Hence it follows that $\theta^{*}(w)=\prod\left\{\theta^{*}(y): y \in \Gamma(w)\right\}$ which divides $\theta\left(w^{\prime}\right)=\prod\left\{\theta(x): x \in \Gamma\left(w^{\prime}\right)\right\}$. Thus $\theta^{*}(w) \geqslant$ $\geqslant \theta\left(w^{\prime}\right)$ in $S$. It is immediate that $w$ covers $w^{\prime}$ in every superior completion $T$. Hence for every such completion $(\Omega, T)$ which preserves suprema, we have

$$
\operatorname{Id}(S, X)=\operatorname{Id}(T, X) .
$$

(5.4.3) We now give an example of a groupoid $(\circ, S)$ such that there exists a suprema preserving superior extension $(\circ, T)$ in which every linear $w^{\prime} \in\langle\circ, X\rangle$ is totally covered in $S$ by $T$, but not every linear $w^{\prime}$ is totally covered in $S$ by $\mathscr{S}(S)$. Let $S$ be the real numbers, trivially ordered. Define

$$
a \circ b=\frac{1}{2}(a+b) \text { for } a, b \in S \text {. }
$$

Let $T=S \cup\{u\}$, where $u$ is adjoined as an element satisfying $a<u$ for all $a \in S$. Then in $(\circ, T)$

$$
a \circ u=u \circ a=u \circ u=u, \text { for all } a \in S,
$$

and $(\circ, T)$ is a suprema preserving superior extension of $(\circ, S)$. Let $w^{\prime}$ be a linear word in $\langle\circ, X\rangle$ and let $w \leqslant w^{\prime}$, say $w=\phi\left(w^{\prime}\right)$. We claim that (5.1.1.1) holds. For suppose that

$$
\left|\theta \phi^{-1} \phi(x)\right|=1 \text { for all } x \in \Gamma\left(w^{\prime}\right)
$$

Then if $\theta^{*} \phi(x) \in \theta \phi^{-1} \phi(x)$, we have $\theta^{*} \phi(x)=\theta \phi^{-1} \phi(x)=\theta(x)$, for $x \in \Gamma\left(w^{\prime}\right)$, whence $\theta\left(w^{\prime}\right)=\theta^{*} \phi\left(w^{\prime}\right)=\theta^{*}(w)$. On the other hand, suppose that

$$
\left|\theta \phi^{-1} \phi(y)\right|>1 \text { for some } y \in \Gamma\left(w^{\prime}\right)
$$

Let $a, b \in \theta \phi^{-1} \phi(y), a \neq b$. Let $\theta^{*} \phi(x) \in \theta \phi^{-1} \phi(x)$ for all $x$ and let $\theta^{*} \phi(y)=a$. Define $\theta_{1}^{*}$ on $\phi(X)$ by $\theta_{1}^{*} \phi(y)=b$, and $\theta_{1}^{*} \phi(x)=\theta^{*} \phi(x)$ otherwise. Then $\theta^{*}(w)=$ $=\theta_{1}^{*}(w)$, whence

$$
\sup _{T}\left\{\theta^{*}(w): \theta^{*} \phi(x) \in \theta \phi^{-1} \phi(x)\right\}=u
$$

It follows that (5.1.1.1) holds, as claimed, and that every linear $w^{\prime} \in\langle\circ, X\rangle$ is totally 
covered in $S$ by $T$. However, $x \circ x \equiv x$ holds in $S$ but not in $\mathscr{S}(S)$, whence by Theorem (5.3) there must exist a linear $w^{\prime} \in\langle\circ, X\rangle$ which is not totally covered in $S$ by $\mathscr{P}(S)$.

\section{(5.5) Examples}

We shall now give two examples of a semigroup $(\circ, S)$, one trivially ordered and one non-trivially ordered, such that $\operatorname{Id}(\Omega, S)=\operatorname{Id}(\Omega, \mathscr{S}(S))$ but $\psi(w) \neq \psi(w)$ for some $w \in\langle\Omega, X\rangle$. In fact, $w$ may be chosen to be $w_{1}$ or $w_{2}$ where $w_{1} \equiv w_{2}$ holds on $(0, S)$.

(5.5.1) Let $S=\{1, a, 0\}$ be the semigroup of (4.23.2) (that is, the nullary operator is now omitted). Then

$$
\operatorname{Id}(S, X)=\operatorname{Id}(\mathscr{P}(S), X)=\mathscr{R}
$$

where $\mathscr{R}$ is given by (4.21.2.1). Let $\psi: X \rightarrow \mathscr{S}(S)$ satisfy $\psi(x)=\{1, a\}$, for some $x \in X$. Then $\psi\left(x^{2}\right)=\psi\left(\left(x^{2}\right) x\right)=\{1, a, 0\}=S$. On the other hand,

and similarly

$$
\begin{aligned}
\psi\left(x^{2}\right) & =\left\{\theta\left(x^{2}\right): \theta(x) \in\{1, a\}\right\} \\
& =\{1,0\}
\end{aligned}
$$

$$
\psi\left(\left(x^{2}\right) x\right)=\{1,0\}
$$

(5.5.2) Let $S=\{1, a, 0\}$ be ordered as in Fig. 2 and let $T=S \cup\{u\},(\cong \mathscr{P}(S))$.

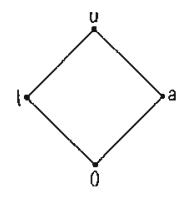

Fig. 2

If $\psi: X \rightarrow T$ with $\psi(x)=u$, then

$$
\psi\left(x^{2}\right)=\psi\left(\left(x^{2}\right) x\right)=u,
$$

while

$$
\psi\left(x^{2}\right)=\psi\left(\left(x^{2}\right) x\right)=1
$$

\section{REFERENCES}

[1] M. N. Bleicher and H. Schneider, Completions of partially ordered sets and universal algebras, Acta Math. Acad. Sci. Hungaricae 17 (1966), 271-301.

[2] M. N. Bleicher, H. Schneider and R. L. Wilson, Permanence of identities on universal algebras, MRC Technical Summary Report \#1339, The University of Wisconsin Mathematics Research Center, 1973. 
[3] A. H. Clifford, Completion of partially ordered semi-groups, presented at Symposium on Semigroup Theory and Applications at Smolenice, Czechoslovakia, June, 1968.

[4] P. M. Cohn, Universal Algebras, Harper Row (1965).

[5] L. Fuchs, On partially ordered algebras I, Colloquium Math. 14 (1966), I15-130.

[6] L. Fuchs, On partially ordered algebras II, Acta Univ. Szegediensis 26 (1965), 34-41.

[7] N.D. Gautam, The Validity of Equations of Complex Algebras, Archiv für Math. Logik und Grundlagenforschung 3 (1957), 117-124.

[8] G. Grätzer, Universal Algebra, Van Nostrand (1968).

[9] R. L. Wilson, Loop isotopism and isomorphism and extensions of Universal Algebras, Thesis, University of Wisconsin, (1969).

University of Wisconsin Madison, Wisconsin

U.S.A. 